\title{
Blastocystis sp. Carriage and Irritable Bowel Syndrome: Is the Association Already Established?
}

\author{
Fernando Salvador ${ }^{1, *}\left(\mathbb{C}\right.$, Beatriz Lobo ${ }^{2}$, Lidia Goterris ${ }^{3} \oplus$, Carmen Alonso-Cotoner ${ }^{2} \oplus$, Javier Santos ${ }^{2}$, \\ Elena Sulleiro $^{3}$, Begoña Bailo ${ }^{4}$, David Carmena ${ }^{4} \mathbb{D}$, Adrián Sánchez-Montalvá ${ }^{1}$, Pau Bosch-Nicolau ${ }^{1}{ }^{1}$, \\ Juan Espinosa-Pereiro ${ }^{1}$, Isabel Fuentes ${ }^{4}$ and Israel Molina ${ }^{1}$
}

1 Department of Infectious Diseases, Vall d'Hebron University Hospital, PROSICS Barcelona, Po Vall d'Hebron 119-129, 08035 Barcelona, Spain; adsanche@vhebron.net (A.S.-M.); pau.boschnicolau@gmail.com (P.B.-N.); macspinosa@gmail.com (J.E.-P.); imolina@vhebron.net (I.M.)

2 Digestive System Research Unit, University Hospital Vall d'Hebron, Centro de Investigación Biomédica en Red de Enfermedades Hepáticas y Digestivas (Ciberehd); Departament de Medicina, Universitat Autònoma de Barcelona, 08193 Cerdanyola del Vallès, Spain; bealoboster@gmail.com (B.L.); 33925mac@gmail.com (C.A.-C.); santosjav@gmail.com (J.S.)

3 Department of Microbiology, Vall d'Hebron University Hospital, PROSICS Barcelona, 08035 Barcelona, Spain; lgoterris@vhebron.net (L.G.); esulleir@vhebron.net (E.S.)

4 National Centre of Microbiology, Instituto de Salud Carlos III, 28220 Madrid, Spain; BEGOBB@isciii.es (B.B.); dacarmena@isciii.es (D.C.); ifuentes@isciii.es (I.F.)

check for updates

Citation: Salvador, F.; Lobo, B.; Goterris, L.; Alonso-Cotoner, C.; Santos, J.; Sulleiro, E.; Bailo, B.; Carmena, D.; Sánchez-Montalvá, A.; Bosch-Nicolau, P.; et al. Blastocystis sp. Carriage and Irritable Bowel Syndrome: Is the Association Already Established?. Biology 2021, 10, 340. https://doi.org/10.3390/ biology10040340

Academic Editors: Anastasios D. Tsaousis, Eleni Gentekaki and Maikel P. Peppelenbosch

Received: 24 February 2021

Accepted: 7 April 2021

Published: 19 April 2021

Publisher's Note: MDPI stays neutral with regard to jurisdictional claims in published maps and institutional affiliations.

Copyright: (C) 2021 by the authors. Licensee MDPI, Basel, Switzerland. This article is an open access article distributed under the terms and conditions of the Creative Commons Attribution (CC BY) license (https:/ / creativecommons.org/licenses/by/ $4.0 /)$.
* Correspondence: fmsalvad@vhebron.net; Tel.: +34-93-274-60-90; Fax: +34-93-489-40-91

Simple Summary: The intestinal protist Blastocystis sp. is one of the most common intestinal parasites observed in humans, and has a worldwide distribution, being more prevalent in developing countries. Although this parasite has been described decades ago, the pathogenic potential it is still not understood completely. It has been suggested that Blastocystis sp. could be related with irritable bowel syndrome, a functional gastrointestinal disorder characterized by abdominal pain, discomfort with defecation, and changes in the frequency or form of stool. In our study, we compare a group of patients with irritable bowel syndrome with a group of healthy volunteers; no differences regarding the occurrence of Blastocystis sp. detection was found between both groups.

Abstract: Background: The aim of the present study is to describe the occurrence of Blastocystis sp. detection among asymptomatic subjects and patients with irritable bowel syndrome in order to evaluate the potential association between irritable bowel syndrome and the parasitic infection. Methods: Cross-sectional study where adult patients with irritable bowel syndrome diagnosed according to Rome IV criteria were included. A control group was formed by asymptomatic subjects older than 18 years. Exclusion criteria were: immunosuppressive condition or having received any drug with demonstrated activity against Blastocystis sp. within the last 6 months before study inclusion. Epidemiological and clinical information was collected from all included participants. Two stool samples were obtained from all participants: one sample for microscopic examination and one sample for Blastocystis sp. PCR detection. Blastocystis sp. infection was defined by the positivity of any of the diagnostic techniques. Results: Seventy-two participants were included (36 asymptomatic subjects and 36 patients with irritable bowel syndrome). Thirty-five (48.6\%) were men, and median age of participants was 34 (IQR 29-49) years. The overall rate of Blastocystis sp. carriage was $27.8 \%(20 / 72)$. The prevalence assessed through microscopic examination was $22.2 \%$ $(16 / 72)$, while the prevalence measured by PCR was $15.3 \%(11 / 72)$. When comparing the presence of Blastocystis sp. between asymptomatic subjects and IBS patients, we did not find any statistically significant difference ( $36.1 \%$ vs. $19.4 \%$ respectively, $p=0.114)$. Conclusions: regarding the occurrence of Blastocystis sp., no differences were found between asymptomatic participants and patients with irritable bowel disease irrespective of the diagnostic technique performed.

Keywords: Blastocystis sp.; irritable bowel syndrome; pathogenesis 


\section{Introduction}

The protist Blastocystis sp. (superphylum Heterokonta) is one of the most common intestinal parasites observed in humans, with up to one billion estimated humans colonized. Blastocystis sp. has a worldwide distribution, being more prevalent in developing countries [1]. This parasite is transmitted by the fecal-oral route, and transmission can occur from human to human or from animal to human [2]. Studies based on the comparison of the nuclear small subunit rRNA gene show that Blastocystis sp. has an extensive molecular diversity; twenty-three subtypes (STs) have been described, although only STs 1-9 and ST12 have been found in humans $[3,4]$.

The pathogenic potential of Blastocystis sp. is a matter of debate since it has been detected in both asymptomatic and symptomatic individuals. When present, clinical features associated with Blastocystis sp. include gastrointestinal symptoms (diarrhea, abdominal pain, vomiting, flatulence, bloating, dyspepsia, and other gastrointestinal symptoms), and cutaneous manifestations such as chronic urticaria [5,6]. Different factors have been suggested to be related with the presence of symptoms, such as the subtype, the parasite load, or factors associated to the host [7-9].

On the one hand, most gastrointestinal symptoms described in Blastocystis sp. carriers are similar to those attributed to irritable bowel syndrome (IBS), a functional gastrointestinal disorder characterized by abdominal pain, discomfort with defecation, and changes in the frequency or form of stool [10]. On the other hand, although the cause of IBS remains unknown, alterations in the gut microbiota including bacteria, parasites, fungi and even viruses have been suggested to be involved. Remarkably, post-infectious gastroenteritis (particularly parasitic infections) has been acknowledged as one of the strongest risk factors for developing IBS [11,12]. There are studies that have suggested a possible link between Blastocystis sp. infection and IBS, while other studies did not observe any association [13-15]. The aim of the present study is to assess the occurrence of Blastocystis sp. among asymptomatic subjects and patients with IBS in order to evaluate the potential association between IBS and the parasitic infection.

\section{Methods}

This is a cross-sectional study performed at Vall d'Hebron University Hospital (Barcelona, Spain) from April 2018 to December 2019. The asymptomatic group (healthy controls) inclusion criteria were: voluntary adult participants (18 years old or above) without gastrointestinal symptoms assessed through a structured and validated questionnaire (the Gastrointestinal Symptom Rating Scale, GSRS) [16]. The symptomatic group inclusion criteria were as follows: adult patients (18 years old or above) with IBS diagnosed according to Rome IV criteria [17]. Exclusion criteria for all participants were: any immunosuppressive condition, having been born outside Spain, having received any drug with demonstrated activity against Blastocystis sp. in the last 6 months before study inclusion (metronidazole, secnidazole, nitazoxanide, cotrimoxazole, paromomycin, iodoquinol, albendazole, and ivermectin), and the presence of cutaneous symptoms compatible with spontaneous chronic urticaria.

Demographic and clinical information was collected from all recruited participants. Moreover, the following epidemiological information was collected through a structured questionnaire: area of residence (urban or rural), regular contact with domestic animals, travelling abroad in the last 12 months or working in close contact with people (e.g., healthcare professionals, teachers, restaurant workers, caregivers). Two stool samples from the same defecation were obtained from all participants: one sample for the microscopic examination and one sample for Blastocystis sp. DNA detection by means of a PolymeraseChain-Reaction (PCR) performed at the Parasitology Reference and Research Laboratory, National Centre for Microbiology (Instituto de Salud Carlos III, Madrid, Spain). Stool samples for Blastocystis sp. DNA detection were refrigerated at $4{ }^{\circ} \mathrm{C}$ until sent to the National Centre for Microbiology (the samples were stored up to two months). Blastocystis 
sp. infection was defined by the positivity of any of the diagnostic techniques. Information regarding the presence of other intestinal parasites was also collected.

\subsection{Statistical and Ethical Issues}

To calculate the sample size a prevalence of $10 \%$ was estimated among asymptomatic participants and 35\% among symptomatic patients based on previous studies [18]. Assuming $5 \%$ of missing data, 41 subjects per group would provide $>80 \%$ power to show significant differences between the two groups (at alpha $<0.05$ ). Categorical data are presented as absolute numbers and proportions, and continuous variables are expressed as medians and interquartile ranges (IQR). The $\chi^{2}$ test or Fisher exact test, when appropriate, was used to compare the distribution of categorical variables, and the Mann-Whitney $U$ test for continuous variables. Results were considered statistically significant if the 2-tailed $p$ value was $<0.05$. SPSS software for Windows (Version 19.0; SPSS Inc, Chicago, IL, USA) was used for statistical analyses.

Procedures were performed in accordance with the ethical standards laid down in the Declaration of Helsinki as revised in 2013, and the study protocol was approved by the Ethical Review Board of the Vall d'Hebron University Hospital (reference number PR (AG) 29/2018). Written informed consent was obtained from all participants.

\subsection{Microbiological Techniques}

During the study period, the diagnosis of intestinal parasites, including Blastocystis sp., was performed by microscopic examination of a fixed and concentrated stool sample using a commercial device (Midi Parasep SF. APACOR. Wokingham, UK). Examination under the microscope was performed on the same day of collection. A sample of approximately $2 \mathrm{~g}$ of stool (size of a pea) is included in the Midi Parasep that is centrifuged at $1500 \mathrm{rpm}$ for $3 \mathrm{~min}$ to decrease the risk of lysis of intestinal protozoa. A mix with methiolate and iodine was used for staining the samples before their examination. Parasite burden was classified as follows: low ( $<1$ parasite/high-power field $\times 400)$, medium (1-5 parasites/high-power field $\times 400)$, and high ( $>5$ parasites / high-power field $\times 400$ ).

For molecular detection, DNA was extracted from $200 \mathrm{mg}$ faecal samples using the QIAmp Fast DNA Stool Mini Kit (QIAGEN, Hilden, Germany). The extracted DNA was stored at $4{ }^{\circ} \mathrm{C}$ until analyses. Identification of Blastocystis sp. was achieved by a direct PCR protocol targeting the small subunit ribosomal RNA (ssu rRNA) gene of the parasite as described elsewhere (18). The assay uses the pan-Blastocystis, barcode primer pair RD5 (5'-GAGCTTTTTAACTGCAACAACG-3') and BhRDr (5'-ATCTGGTTGATCCTGCCAGT$\left.3^{\prime}\right)$ to amplify a PCR product of $\sim 600 \mathrm{bp}$. Amplification reactions $(25 \mu \mathrm{L})$ included $5 \mu \mathrm{L}$ of template DNA and $0.5 \mu \mathrm{M}$ of each primer, 2.5 units of MyTAQ ${ }^{\mathrm{TM}}$ DNA polymerase (Bioline $\mathrm{GmbH}$, Luckenwalde, Germany), and $5 \times \mathrm{MyTAQ}^{\mathrm{TM}}$ Reaction Buffer containing $5 \mathrm{mM}$ dNTPs and $15 \mathrm{mM} \mathrm{MgCl}_{2}$. Amplification conditions consisted of one step of $95^{\circ} \mathrm{C}$ for $3 \mathrm{~min}$, followed by 30 cycles of $1 \mathrm{~min}$ each at 94,59 , and $72{ }^{\circ} \mathrm{C}$, with an additional $2 \mathrm{~min}$ final extension at $72{ }^{\circ} \mathrm{C}$. Negative and positive controls were included in every PCR run. Obtained amplicons were separated by electrophoresis on 2\% D5 agarose gels stained with Pronasafe (Conda, Madrid, Spain).

For sequence analysis, amplicons of the expected size were directly sequenced in both directions using the RD5/BhRDr primer pair in $10 \mu \mathrm{L}$ reactions. DNA sequencing was conducted by capillary electrophoresis using the BigDye ${ }^{\circledR}$ Terminator chemistry (Applied Biosystems Foster City, CA, USA) on an ABI PRISM 3130 automated DNA sequencer. Raw sequences were examined with Chromas Lite version 2.1 software (http:/ / chromaslite. software.informer.com/2.1, accessed on 1 February 2021) to generate consensus sequences. These sequences were compared with reference sequences deposited at the National Centre for Biotechnology Information (NCBI) using the BLAST tool (http:/ /blast.ncbi.nlm.nih. gov/Blast.cgi, accessed on 1 February 2021) to confirm that they belong to Blastocystis. Subtype and allele calling was carried out at the Blastocystis 18S database (http:/ / pubmlst. org/blastocystis/, accessed on 1 February 2021). Blastocystis sequences generated in the 
present study were deposited in the GenBank public repository database under accession numbers MT913015 and MT913016.

\section{Results}

Overall, 89 subjects were offered to participate in the study (41 healthy controls and 48 patients diagnosed of IBS-diarrhea). Five controls and 12 cases did not provide the stool samples and were excluded from the study; therefore, 36 controls and 36 cases were included in the analysis (see Figure 1). Thirty-five out of $72(48.6 \%)$ were men and median age of participants was 34 (IQR 29-49) years. Regarding epidemiological factors, 4 (5.6\%) participants were living in a rural environment, $32(44.4 \%)$ had regular contact with animals, $30(41.7 \%)$ were working in close contact with people, and $34(47.2 \%)$ had travelled during the last year. Comparison of both groups regarding epidemiological factors is summarized in Table 1.

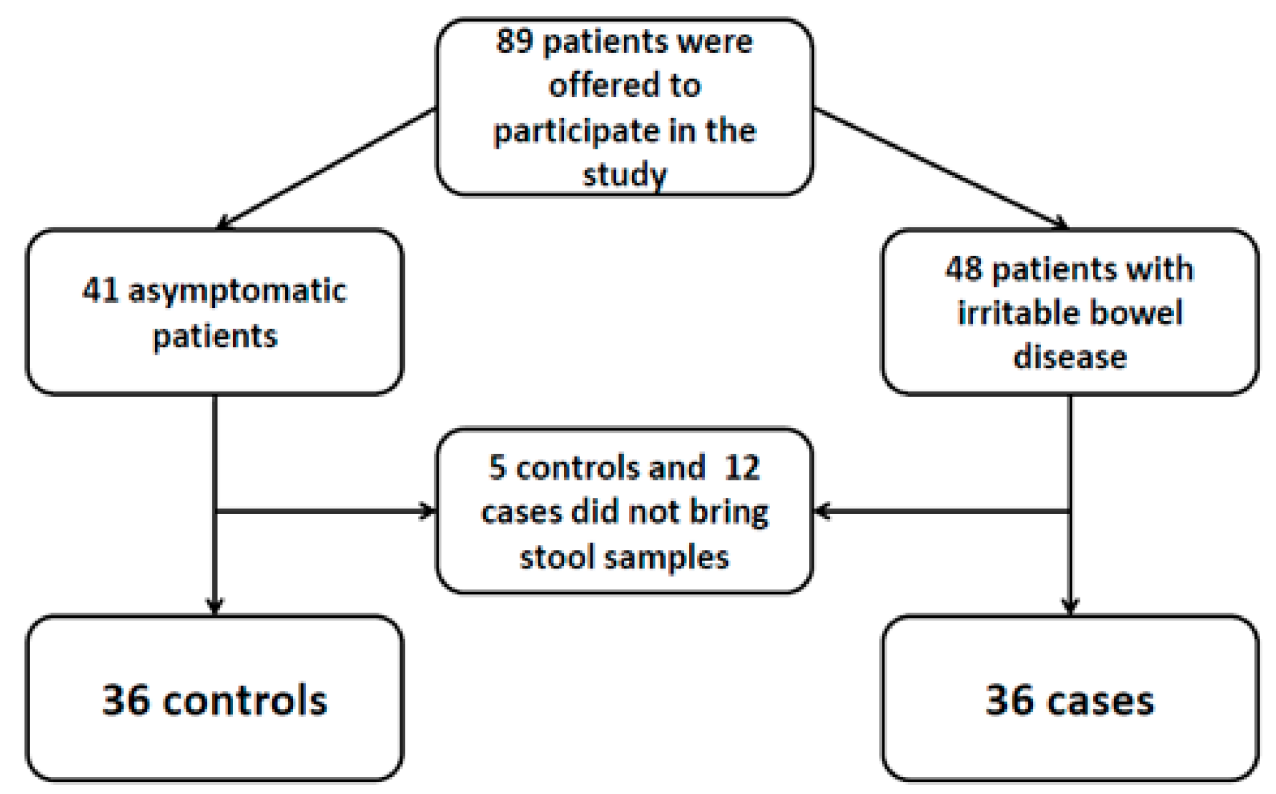

Figure 1. Flow diagram of patients.

Table 1. Comparison of epidemiological characteristics between healthy controls and patients with IBS.

\begin{tabular}{cccc}
\hline & Healthy Controls $(\mathbf{n}=36)$ & IBS patients $(\mathbf{n}=\mathbf{3 6})$ & $p$ Value \\
\hline Age, years & $30(29-38.7)$ & $43(29.5-50.5)$ & 0.094 \\
Gender, male & $19(52.8 \%)$ & $16(44.4 \%)$ & 0.479 \\
Living in rural environment & $3(8.3 \%)$ & $1(2.8 \%)$ & 0.614 \\
Regular contact with animals & $11(30.6 \%)$ & $21(58.3 \%)$ & 0.018 \\
Travelling abroad in the last 12 months & $26(72.2 \%)$ & $8(22.2 \%)$ & $<0.001$ \\
Travelling to low or middle income & $14(38.8 \%)$ & $1(2.7 \%)$ & $<0.001$ \\
country in the last 12 months & $20(55.6 \%)$ & $10(27.8 \%)$ & 0.017 \\
Working in close contact with people & & \\
\hline
\end{tabular}

Note. Data are reported as number (\%) of patients or median (IQR).

The overall occurrence of Blastocystis sp. detection was $27.8 \%$ (20/72). The positive rate assessed through microscopic examination was $22.2 \%(16 / 72)$, while the positive rate measured by PCR was $15.3 \%(11 / 72)$. Regarding the parasite burden among subjects with positive microscopic examination, in $6(37.5 \%)$ patients it was low, in $6(37.5 \%)$ patients it was medium, and in $4(25 \%)$ it was high. Detection of concomitant intestinal microbial eukaryotes was only observed in four (5.6\%) participants: two cases of Endolimax nana, one case of Dientamoeba fragilis, and one case of Entamoeba hartmanni. When comparing the occurrence of Blastocystis sp. detection between the two groups (irrespectively of the 
diagnostic technique performed), we did not find any statistically significant difference (see Table 2).

Table 2. Microbiological results among healthy controls and patients with IBS.

\begin{tabular}{|c|c|c|c|}
\hline & Healthy Controls $(n=36)$ & $\begin{array}{c}\text { IBS Patients }(\mathrm{n}= \\
36)\end{array}$ & $p$ Value \\
\hline Blastocystis sp. detection (microscopy + PCR) & $13(36.1 \%)$ & $7(19.4 \%)$ & 0.114 \\
\hline $\begin{array}{l}\text { Blastocystis sp. detection by microscopic examination } \\
\text { Parasite burden }(\mathrm{n}=16)^{*}\end{array}$ & $10(27.8 \%)$ & $6(16.7 \%)$ & 0.257 \\
\hline Low $(<1$ parasite/high-power field × $\times 400)$ & $4 / 10(40 \%)$ & $2 / 6(33.3 \%)$ & 1 \\
\hline Medium $(1-5$ parasite/high-power field $\times 400)$ & $4 / 10(40 \%)$ & $2 / 6(33.3 \%)$ & 1 \\
\hline High $(>5$ parasite/high-power field $\times 400)$ & $2(20 \%)$ & $2 / 6(33.3 \%)$ & 0.604 \\
\hline Positive Blastocystis sp. PCR & $8(22.2 \%)$ & $3(8.3 \%)$ & 0.101 \\
\hline Detection of other intestinal microbial eukaryotes & $1(2.8 \%)$ & $3(8.3 \%)$ & 0.614 \\
\hline
\end{tabular}

Note. Data are reported as number (\%) of patients. * Among patients with positive microscopic examination.

The Blastocystis sp. subtypes and alleles could only be determined by Sanger sequencing in two subjects, both of them belonging to the asymptomatic group: a 29-year old woman with high parasitic burden in the microscopic examination (ST1, allele 4), and a 54-year old woman with negative microscopic examination (ST6, allele 122).

\section{Discussion}

Here we compare the results of 36 asymptomatic healthy controls and 36 patients with IBS through microscopic examination of stool samples and PCR in order to detect Blastocystis sp. We did not find any statistically significant difference between both groups regarding the occurrence of Blastocystis sp. detection irrespectively of the diagnostic technique performed. Taking into account both detection methods (microscopic examination and PCR), the frequency of Blastocystis sp. identification among our study population was $27.8 \%$. This rate is similar to those observed in other Spanish studies performed among adult patients, ranging from $17.3 \%$ to $35.2 \%$ [9,19,20]. However, Spanish studies performed among children showed prevalence slightly lower than in adults, ranging from $13 \%$ to $15 \%$ [21-23]. Age-related patterns for Blastocystis sp. carriage have been previously demonstrated both in developed and developing countries [24-26].

Surprisingly, the percentage of subjects with a positive Blastocystis-positive result by PCR was lower (15.3\%) than that observed by microscopic examination $(22.2 \%)$. PCR techniques for diagnosis of intestinal parasitic infections are usually more sensitive than classical parasitological techniques, although the last ones are highly observer-dependant, and sensitivity can vary widely from eye to eye [27]. Moreover, it is important to note that the great majority of positive cases by microscopic examination had a low or medium parasite burden (75\%), which could explain the low percentage of positive cases detected by PCR. Suboptimal preservation of fecal samples and insufficient removal of PCR inhibitors during DNA extraction and purification may also account for the failure of PCR amplification reactions. The difference in the amount of stool sample analyzed must be also taken into account (2 $\mathrm{g}$ for microscopy versus $200 \mathrm{mg}$ for PCR).

After decades of research, the pathogenic potential of Blastocystis sp. is not well understood [5]. The fact that the gastrointestinal symptoms in patients presenting with Blastocystis sp. in feces are very unspecific; the high percentage of asymptomatic carriers, and the coexistence of other possible causes of the symptoms (e.g., other viral, bacterial or parasitic agents), makes it very difficult to attribute the clinical symptoms to the presence of Blastocystis sp. Regarding IBS, contradictory results have been observed. One recent example is the one described by Shafiei et al., where 100 patients with IBS and 100 asymptomatic subjects were studied, with higher percentage of Blastocystis sp. carriage among IBS patients than in asymptomatic controls ( $15 \%$ vs. $6 \%$, respectively) [28]. In contrast, Krogsgaard et al. described a higher proportion of Blastocystis sp. detection among control 
subjects (22\%) compared to the one found in IBS patients (15\%) [15]. A recent systematic review and meta-analysis that explored the role of Blastocystis sp. and Dientamoeba fragilis in IBS showed that individuals with Blastocystis sp. carriage were found to have a positive association with IBS (OR, 2.19; 95\% CI, 1.54-3.13), while this association was not observed for $D$. fragilis carriage (OR, 1.13; 95\% CI, 0.22-5.72) [29].

In our study population, percentage of Blastocystis sp. detection was higher in asymptomatic healthy controls than in IBS patients, although the difference was not statistically significant. A study performed in Malian children showed that Blastocystis-colonized children had higher gut microbiota diversity than Blastocystis-noncolonized children [30]. Indeed, some authors have regarded Blastocystis sp. as a constituent of the healthy gut microbiota [31]. High gut microbiota diversity is usually considered a healthy gut sign, since it decreases the possibility of having potentially pathogenic bacteria in the human gut. This fact could explain the results of the studies that show higher proportion of Blastocystis sp. carriage among healthy controls.

Another aspect that has been related to IBS is the Blastocystis subtype. A study performed among senior high school students in Indonesia observed an association between Blastocystis ST1 and irritable bowel syndrome-diarrhea [32]. Moreover, in the previously mentioned systematic review, considering the subtypes, meta-analysis results showed significant positive association for ST1 (OR, 4.40; 95\% CI, 2.81-6.90) and ST3 (OR, 1.94; 95\% CI, 1.36-2.77) to be potential risk factors for IBS [29]. Unfortunately, sequencing for ST detection could only be achieved in two participants of our cohort.

It has been suggested that parasite burden could be associated with the presence of clinical symptoms in patients with Blastocystis sp. detection, especially in those having $>5$ parasites/high-power field $(\times 400)$ [2]. In our study, we did not observe any difference regarding the parasite burden between the two groups. This finding is in agreement with the results of other studies, such as the ones observed in the study published by VargasSánchez et al., where 50 asymptomatic and 50 IBS patients with Blastocystis sp. detection were evaluated, and similar parasitological loads between the two groups were observed measured by quantitative PCR [33].

It is important to note that, when comparing epidemiological data between both groups in our study, the healthy group control had a higher proportion of subjects having travelled abroad in the last year before inclusion compared with the IBS patients group ( $72.2 \%$ versus $22.2 \%$ respectively, $p<0.001)$. A statistically significant difference remains when we only take into account travels to low- or middle-income countries. Recent travel has previously been described as a risk factor for Blastocystis sp. carriage, and could be a confounder when interpreting the results [9].

We acknowledge some limitations for our study. Firstly, as it is a cross-sectional study, we only have evaluated the stool samples from participants at one single time; it is well known that the gut microbiota changes over time. Another limitation of the study is that STs and alleles of positive cases could not be sequenced in most of the participants; hence the presence of IBS could not be related to specific STs (see more information in Appendix A).

\section{Conclusions}

In conclusion, the overall occurrence of Blastocystis sp. detection in our study was $27.8 \%$, but no statistically significant differences were found between asymptomatic healthy controls and patients with IBS irrespective of the diagnostic technique performed (microscopic examination, PCR or both). Further studies are needed in order to establish the pathogenic potential of Blastocystis sp.

Author Contributions: F.S., E.S. and I.M. planned and designed the study; F.S., B.L., C.A.-C., J.S., A.S.-M., P.B.-N., J.E.-P. and I.M. included the patients and collected the data; L.G., E.S., B.B., D.C. and I.F. performed the microbiological techniques; F.S. analyzed the data and wrote the first draft of the manuscript. All authors have read and agreed to the published version of the manuscript. 
Funding: This research received no specific grant from any funding agency in the public, commercial, or not-for-profit sectors.

Institutional Review Board Statement: The study was conducted according to the guidelines of the Declaration of Helsinki, and approved by the Ethics Committee) of Vall d'Hebron University Hospital (protocol code PR (AG) 29/2018), approved on April 2018).

Informed Consent Statement: Written informed consent was obtained from all participants.

Data Availability Statement: All data generated or analysed during this study are included in this published article.

Acknowledgments: This study was supported by the ISCIII-Collaborative Research Network on Tropical Diseases (RICET) and the European Regional Development Fund (ERDF): RD16/0027/0003 and RD16CIII/0003/0004.

Conflicts of Interest: The authors declare that they have no competing interest.

Ethics Approval and Consent to Participate: The study protocol was approved by the Ethical Review Board of the Vall d'Hebron University Hospital (reference number PR (AG) 29/2018). Written informed consent was obtained from all participants.

\section{Appendix A}

During the peer-revision process some concerns regarding the PCR technique raised. Inhibition controls are normally used in real-time PCR protocols. In our case, we took advantage of a robust, widely-used PCR protocol for the specific amplification of the ssu rRNA gene of Blastocystis sp. using pan-Blastocystis primers. Although we cannot completely ruled out that some of our false-negative results by ssu-PCR were the consequence of inhibition of the amplification reaction, we believe that the main problem was the long storage period (up to 2 months at $4 \mathrm{C}$ ) of the faecal material, that very likely has compromised the quality of the purified DNA and the diagnostic performance of the PCR. This point has been conveniently explained in the discussion section of the manuscript as a limitation of the study. Most of the ssu-PCR amplicons of the expected size generated faint bands on gel electrophoresis. In Sanger sequencing these bands generated poor-quality sequences that, although belonging to Blastocystis sp., did not allow the assignment to a specific subtype or allele calling. Therefore, we can confirm that those bands did not correspond to unspecific amplification reactions.

\section{References}

1. Fletcher, S.M.; Stark, D.; Harkness, J.; Ellis, J.T. Enteric protozoa in the developed world: A public health perspective. Clin. Microbiol. Rev. 2012, 25, 420-449. [CrossRef]

2. Tan, K.S.W. New insights on classification, identification, and clinical relevance of Blastocystis spp. Clin. Microbiol. Rev. 2008, 21, 639-665. [CrossRef]

3. Stensvold, C.R.; Clark, C.G. Pre-empting Pandora's Box: Blastocystis Subtypes Revisited. Trends Parasitol. 2020, 36, $229-232$. [CrossRef]

4. Maloney, J.G.; Lombard, J.E.; Urie, N.J.; Shivley, C.B.; Santin, M. Zoonotic and genetically diverse subtypes of Blastocystis in US pre-weaned dairy heifer calves. Parasitol. Res. 2019, 118, 575-582. [CrossRef] [PubMed]

5. Roberts, T.; Stark, D.; Harkness, J.; Ellis, J. Update on the pathogenic potential and treatment options for Blastocystis sp. Gut Pathog. 2014, 6, 17. [CrossRef] [PubMed]

6. Salvador, F.; Sulleiro, E.; Sánchez-Montalvá, A.; Alonso, C.; Santos, J.; Fuentes, I.; Molina, I. Epidemiological and clinical profile of adult patients with Blastocystis hominis infection in Barcelona, Spain. Parasites Vectors 2016, 9, 1-7. [CrossRef] [PubMed]

7. Roberts, T.; Stark, D.; Harkness, J.; Ellis, J. Subtype distribution of Blastocystis isolates identified in a Sydney population and pathogenic potential of Blastocystis. Eur. J. Clin. Microbiol. Infect. Dis. 2012, 32, 335-343. [CrossRef] [PubMed]

8. Kaya, S.; Cetin, E.S.; Aridogan, B.C.; Arikan, S.; Demirci, M. Pathogenicity of Blastocystis hominis, a clinical reevaluation. Turk. J. Parasitol. 2007, 31, 184-187.

9. Hidalgo, L.; Salvador, F.; Sulleiro, E.; López, I.; Balladares, M.; García, E.; Paz, C.; Sánchez-Montalvá, A.; Bosch-Nicolau, P.; Sao-Avilés, A.; et al. Evaluation of risk factors associated to detection of Blastocystis sp in fecal samples in population from Barcelona, Spain: A case-control study. Eur. J. Clin. Microbiol. Infect. Dis. 2019, 38, 1241-1247. [CrossRef]

10. Lacy, B.E.; Mearin, F.; Chang, L.; Chey, W.D.; Lembo, A.J.; Simren, M.; Spiller, R. Bowel disorders. Gastroenterology. 2016, 150 , 1393-1407. [CrossRef] [PubMed] 
11. Botschuijver, S.; Roeselers, G.; Levin, E.; Jonkers, D.M.; Welting, O.; Heinsbroek, S.E.; de Weerd, H.H.; Boekhout, T.; Fornai, M.; Masclee, A.A.; et al. Intestinal fungal dysbiosis is associated with visceral hypersensitivity in patients with irritable bowel syndrome and rats. Gastroenterology 2017, 153, 1026-1039. [CrossRef]

12. Klem, F.; Wadhwa, A.; Prokop, L.J.; Sundt, W.J.; Farrugia, G.; Camilleri, M.; Singh, S.; Grover, M. Prevalence, risk factors, and outcomes of irritable bowel syndrome after infectious enteritis: A systematic review and meta-analysis. Gastroenterology 2017, 152, 1042-1054. [CrossRef] [PubMed]

13. Jimenez-Gonzalez, D.E.; Martinez-Flores, W.A.; Reyes-Gordillo, J.; Ramirez-Miranda, M.E.; Arroyo-Escalante, S.; RomeroValdovinos, M.; Stark, D.; Souza-Saldivar, V.; Martinez-Hernandez, F.; Flisser, A.; et al. Blastocystis infection is associated with irritable bowel syndrome in a Mexican patient population. Parasitol. Res. 2011, 110, 1269-1275. [CrossRef]

14. Yakoob, J.; Beg, M.A.; Zaman, V.; Jafri, W.; Jafri, N.; Khan, R.; Islam, M. Irritable bowel syndrome: In search of an etiology: Role of Blastocystis hominis. Am. J. Trop. Med. Hyg. 2004, 70, 383-385. [CrossRef]

15. Krogsgaard, L.R.; Engsbro, A.L.; Stensvold, C.R.; Nielsen, H.V.; Bytzer, P. The prevalence of intestinal parasites is not greater among individuals with irritable bowel syndrome: A population-based case-control study. Clin. Gastroenterol. Hepatol. 2015, 13, 507-513. [CrossRef]

16. Kulich, K.R.; Madisch, A.; Pacini, F.; Piqué, J.M.; Regula, J.; Van Rensburg, C.J.; Újszászy, L.; Carlsson, J.; Halling, K.; Wiklund, I.K. Reliability and validity of the Gastrointestinal Symptom Rating Scale (GSRS) and Quality of Life in Reflux and Dyspepsia (QOLRAD) questionnaire in dyspepsia: A six-country study. Health Qual. Life Outcomes 2008, 6, 12. [CrossRef] [PubMed]

17. Mearin, F.; Rey, E.; Balboa, A. Functional and motor gastrointestinal disorders. Gastroenterol. Hepatol. 2016, 39 (Suppl. 1), 3-13. [CrossRef]

18. Scicluna, S.M.; Tawari, B.; Clark, C.G. DNA barcoding of Blastocystis. Protist 2006, 157, 77-85. [CrossRef] [PubMed]

19. Not, A.; Salvador, F.; Goterris, L.; Sulleiro, E.; López, I.; Balladares, M.; García, E.; Paz, C.; Sánchez-Montalvá, A.; Bosch-Nicolau, P.; et al. Microscopic examination after concentration techniques for Blastocystis sp. detection in serial faecal samples: How many samples are needed? Parasite Epidemiol. Control. 2020, 9, e00137. [CrossRef] [PubMed]

20. Paulos, S.; Köster, P.C.; De Lucio, A.; Hernández-De-Mingo, M.; Cardona, G.A.; Fernández-Crespo, J.C.; Stensvold, C.R.; Carmena, D. Occurrence and subtype distribution of Blastocystis sp. in humans, dogs and cats sharing household in northern Spain and assessment of zoonotic transmission risk. Zoonoses Public Health 2018, 65, 993-1002. [CrossRef]

21. Muadica, A.S.; Köster, P.C.; Dashti, A.; Bailo, B.; Hernández-de-Mingo, M.; Reh, L.; Balasegaram, S.; Verlander, N.Q.; Ruiz Chércoles, E.; Carmena, D. Molecular diversity of Giardia duodenalis, Crysptosporidium spp. and Blastocystis sp. in asymptomatic school children in Leganés, Madrid (Spain). Microorganisms 2020, 8, 466. [CrossRef]

22. Reh, L.; Muadica, A.S.; Köster, P.C.; Balasegaram, S.; Verlander, N.Q.; Chércoles, E.R.; Carmena, D. Substantial prevalence of enteroparasites Cryptosporidium spp., Giardia duodenalis and Blastocystis sp. in asymptomatic schoolchildren in Madrid, Spain, November 2017 to June 2018. Eurosurveillance 2019, 24, 1900241.

23. Soriano, J.M.; Domènech, G.; Martínez, M.C.; Mañes, J.; Soriano, F. Intestinal parasitic infections in hosted Saharawi children. Trop. Biomed. 2011, 28, 557-562.

24. El Safadi, D.; Cian, A.; Nourrisson, C.; Pereira, B.; Morelle, C.; Bastien, P.; Bellanger, A.P.; Botterel, F.; Candolfi, E.; Desoubeaux, G.; et al. Prevalence, risk factors for infection and subtype distribution of the intestinal parasite Blastocystis sp. from a large-scale multi-center study in France. BMC Infect. Dis. 2016, 16, 451. [CrossRef] [PubMed]

25. Pipatsatitpong, D.; Rangsin, R.; Leelayoova, S.; Naaglor, T.; Mungthin, M. Incidence and risk factors of Blastocystis infection in an orphanage in Bangkok, Thailand. Parasites Vectors 2012, 5, 37. [CrossRef] [PubMed]

26. Muadica, A.S.; Balasegaram, S.; Beebeejaun, K.; Köster, P.C.; Bailo, B.; Hernández-De-Mingo, M.; Dashti, A.; Dacal, E.; Saugar, J.M.; Fuentes, I.; et al. Risk associations for intestinal parasites in symptomatic and asymptomatic schoolchildren in central Mozambique. Clin. Microbiol. Infect. 2020. [CrossRef] [PubMed]

27. Suresh, K.; Smith, H. Comparison of methods for detecting Blastocystis hominis. Eur. J. Clin. Microbiol. Infect. Dis. 2004, 23, 509-511. [CrossRef]

28. Shafiei, Z.; Esfandiari, F.; Sarkari, B.; Rezaei, Z.; Fatahi, M.R.; Asl, S.M.K.H. Parasitic infections in irritable bowel syndrome patients: Evidence to propose a possible link, based on a case-control study in the south of Iran. BMC Res. Notes 2020, 13, 264. [CrossRef]

29. Rostami, A.; Riahi, S.M.; Haghighi, A.; Saber, V.; Armon, B.; Seyyedtabaei, S.J. The role of Blastocystis sp. and Dientamoeba fragilis in irritable bowel syndrome: A systematic review and meta-analysis. Parasitol. Res. 2017, 116, 2361-2371. [CrossRef]

30. Kodio, A.; Coulibaly, D.; Koné, A.K.; Konaté, S.; Doumbo, S.; Guindo, A.; Bittar, F.; Gouriet, F.; Raoult, D.; Thera, M.A.; et al. Blastocystis colonization is associated with increased diversity and altered gut bacterial communities in healthy malian children. Microorganisms 2019, 7, 649. [CrossRef]

31. Tito, R.Y.; Chaffron, S.; Caenepeel, C.; Lima-Mendez, G.; Wang, J.; Vieira-Silva, S.; Falony, G.; Hildebrand, F.; Darzi, Y.; Rymenans, L.; et al. Population-level analysis of Blastocystis subtype prevalence and variation in the human gut microbiota. Gut 2019, 68, 1180-1189. [CrossRef] [PubMed]

32. Kesuma, Y.; Firmansyah, A.; Bardosono, S.; Sari, I.P.; Kurniawan, A. Blastocystis ST-1 is associated with irritable bowel syndromediarrhoea (IBS-D) in Indonesian adolescences. Par. Epid. Cont. 2019, 6, e112. [CrossRef] [PubMed]

33. Vargas-Sánchez, G.B.; Romero-Valdovinos, M.; Ramírez-Guerrero, C.; Vargas-Hernández, I.; Ramírez-Miranda, M.E.; MartínezOcaña, J.; Valadez, A.; Ximenez, C.; Lopez-Escamilla, E.; Hernandez-Campos, M.E.; et al. Blastocystis isolates from patients with irritable bowel syndrome and from asymptomatic carriers exhibit similar parasitological loads, but significantly different generation times and genetic variability across multiple subtypes. PLoS ONE 2015, 10, e0124006. [CrossRef] [PubMed] 